\title{
SiPMs - A revolution for high dynamic range applications
}

\author{
T. Bretz*, J. Kemp, L. Middendorf, C. Peters, J. Schumacher \\ III. Physikalisches Institut A, RWTH Aachen University, Germany \\ Corresponding authors: \\ tbretz@physik.rwth-aachen.de \\ kemp@physik.rwth-aachen.de \\ schumacher@physik.rwth-aachen.de
}

\section{R. Engel, R. Smida, D. Veberic}

Institute for Nuclear Physics, Karlsruhe Institute of Technology, Germany

In a wide range of applications, semi-conductor photo sensors (SiPMs) are increasingly replacing classical photo multiplier tubes (PMT). They have the advantage of an easier handling due to their significantly lower bias voltage and a long life time without aging. Usually, detectors need an adapted design for the application of SiPMs due to their smaller size compared to PMTs.

While the linear dynamic range of a PMT is inherently limited and usually depends strongly on the individual PMTs, SiPMs promise a dynamic range which only depends on the SiPM type applied and not on the individual sensor.

SiPMs are compiled from individual Avalanche Photo Diodes operated in Geiger-mode (G-APD). Every of these diodes is only capable of the detection of a single photon at a time. Thus, the number of G-APDs inherently limits the dynamic range of a SiPM. Strictly speaking, a SiPM is non-linear starting from the first detected photon. If this non-linearity is taken into account, the dynamic range for today's sensors can reach $10^{6}$ for coincident photons. A complication arises for extended pulses from the fact that typical re-charge times of individual cells are in the order of several nano-second.

With a $3.8 \mathrm{~m}^{2}$ scintillator detector developed for the upgrade of the Pierre-Auger Observatory, it has been shown that SiPMs can nowadays act as an ideal replacement even in applications which require a high dynamic range. This has been successfully proven by operating two identical detectors on top of each other, one read out with SiPMs and one by a PMT. It is demonstrated that even at very strong illumination the SiPM response is still understood. Furthermore, laboratory measurements confirm that individual sensors are, within the systematic errors, exhibiting identical response. Given the precision of the devices and their advantages in operation, including the possibility of characterizing their response during measurement without any additional calibration device, the application of SiPMs will be a revolution for high dynamic-range applications, significantly reducing systematic uncertainties due to improved stability.

35th International Cosmic Ray Conference - ICRC2017

10-20 July, 2017

Bexco, Busan, Korea

*Speaker. 


\section{The SiPM Module}

A dedicated SiPM module was developed for applications in scintillator detectors, see Fig. 1. The module contains two SiPMs (Hamamatsu S13360-6025PE [1]) with an area of $6 \times 6 \mathrm{~mm}^{2}$ each and a cell pitch of $25 \mu \mathrm{m}$, thus hosting 115,200 cells. The power is supplied by a Hamamatsu integrated circuit (C11204-02 [2]), which ensures that both SiPMs are always operated at the same overvoltage even under varying temperature. For each module, two SiPMs with breakdown voltages as identical as possible were selected. Communication and power is realized through a USB connection.

The signal of both SiPMs is summed-up and split into three different amplifiers. A channel with very high gain allows for measurements with single Photoelectron-Equivalent (PE) resolution. A high gain channel is optimized to resolve a signal of a few ten $\mathrm{PE}$ as for example expected from a Minimum Ionizing Particle (MIP) in a scintillator. A Low Gain (LG) channel allows to exploit the full dynamic range of the SiPMs.

For a good optical coupling, a light guiding cone is glued onto each SiPM. Its design is based on a classical Winston cone [3]. The glue (Epo-Tek 310 M-2 [4]) has a refractive index between that of the cones and the SiPM surface to ensure good transmission.

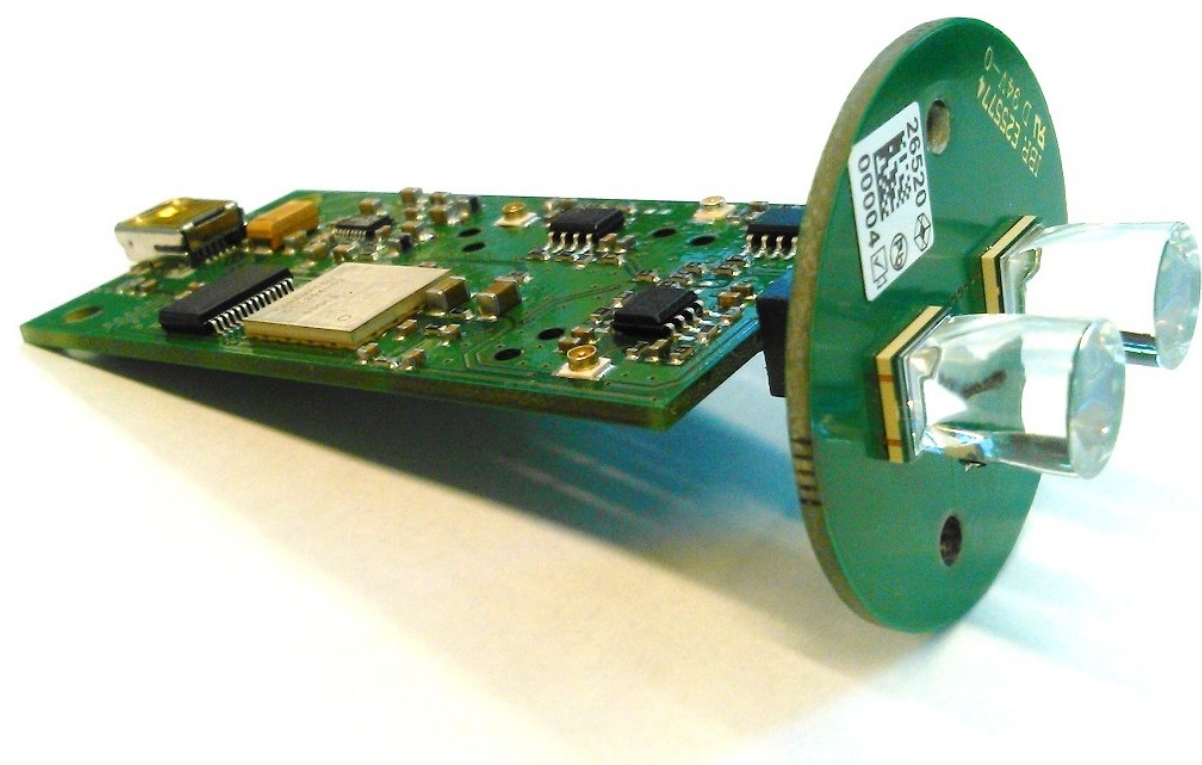

Figure 1: The SiPM module used to measure the SiPM's dynamic range.

\section{Comparison of two SiPMs}

Today's semiconductors are a high-precision mass product. To demonstrate that the response of two devices of the same type is nearly identical, they are exposed to the same light flux. The active area of two SiPMs was placed on a printed circuit board (PCB) at the same distance from the center with high mechanical resolution (c.f. Fig. 1). The SiPMs are operated with the same voltage but read-out individually. A LED is pulsed with programmable amplitude and a constant 

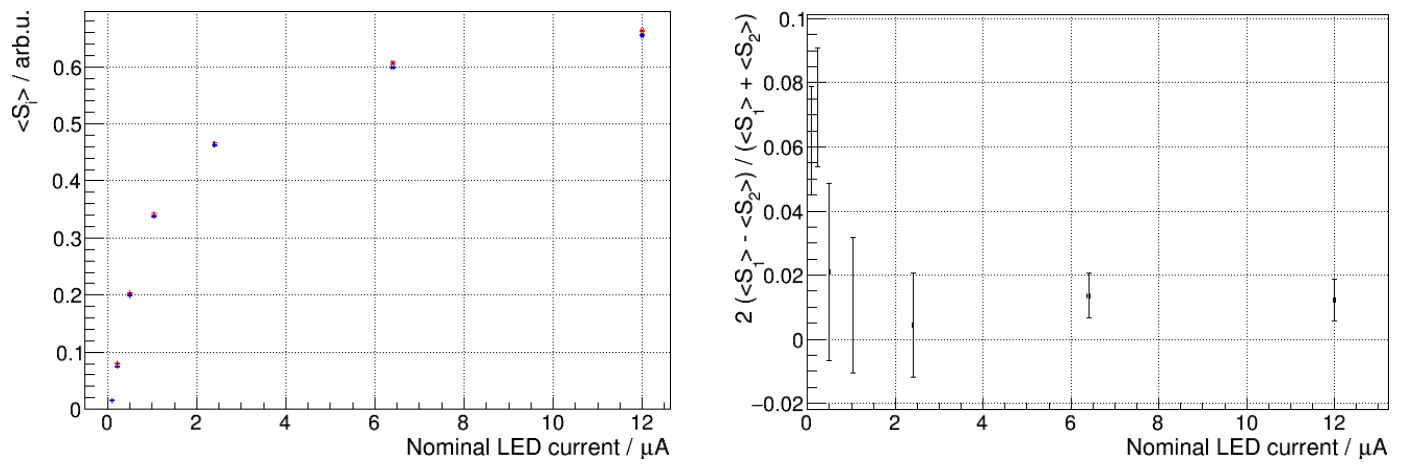

Figure 2: The individual SiPM response (left) and the full relative difference $\Delta S / \bar{S}$ (right) of the two SiPMs vs. the light flux of the LED. The difference in the low-light flux region below $2 \mu \mathrm{A}$ is dominated by Poissonian fluctuations and the difference above $4 \mu \mathrm{A}$ can be explained by the difference in gain of the two SiPMs, see text.

pulse width of less than $10 \mathrm{~ns}$, and the PCB is rotated around its center. After $180^{\circ}$ the active areas of the SiPMs have switched places in the light beam. For an inhomogeneous light beam, a sine dependency with a frequency of one revolution is expected. The average offset from zero is then due to the difference in the response of the two SiPM and the remaining systematic effects, mainly the precision with which the rotation axis coincides with the center between the two SiPMs. A mechanically robust setup has been built with commercially available high-temperature drainage pipes. Re-assembling the complete setup and repeating the measurement several times gives a handle on the systematic error which has been estimated to be $0.4 \%$.

Fig. 2 (left) shows a linear sample measurement comparing the response of the two SiPMs as a function of the nominal LED current which is proportional to the number of incident photons. As the breakdown-voltage of the two SiPMs is not identical, and the same absolute voltage is applied, the applied overvoltage deviates by $0.6 \%$. Thus, a gain difference of $0.6 \%$ is expected. It can be seen that the number of detected photons per pulse is far in the non-linear region of the SiPMs even though the SiPMs are composed of 57,600 cells. Difference in the response of the two SiPMs are small as can be seen in Fig. 2 (right). This plot shows the full relative difference $(\Delta S / \bar{S})$ of the two SiPMs versus the LED current. After re-assembling the setup multiple times $\Delta S / \bar{S}$ at $12 \mu \mathrm{A}$ becomes $(0.7 \pm 0.4) \%$. This is consistent with the difference in the gain of $0.6 \%$ of the two SiPMs as mentioned above.

Although only two SiPMs have been compared, for these two devices no deviation from the expected behavior has been found even at highly-saturating light fluxes. Measurements of more devices are planned for the near future.

\section{Setup for SiPM and PMT comparison}

The dynamic range of the SiPMs should be explored in a realistic setup measuring cosmic-ray initiated air-showers. In addition, the recovery of signals measured in the highly non-linear regime of the SiPM response is studied. In this setup, this corresponds to more than $\sim 1000$ MIP.

Two identical scintillator detectors with an effective detection area of $\sim 3.8 \mathrm{~m}^{2}$, designed for an upgrade of the Pierre Auger Observatory [5, 6], are stacked on top of each other and used to 


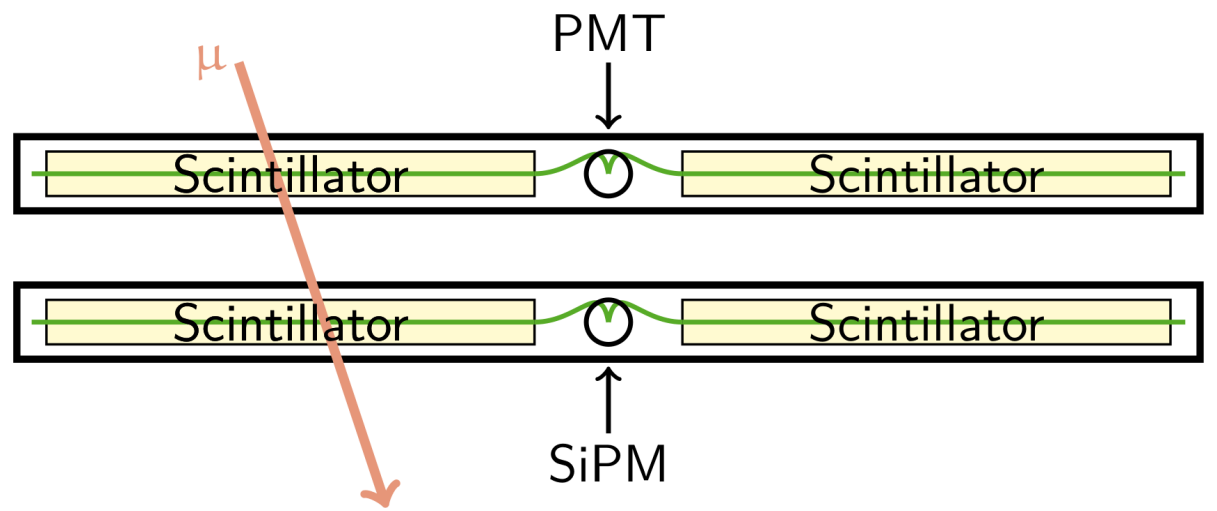

Figure 3: A sketch of the measurement setup. The top scintillator detector is equipped with a PMT, the bottom one with a SiPM module.

measure the same air showers. The top detector is equipped with a high-linearity PMT (Hamamatsu R9420 [7]) used as a reference for the signal and the bottom detector is equipped with one SiPM module as described in Sec. 1. A sketch of this setup is shown in Fig. 3.

The readout of the signals is performed with a Picoscope [8] with four analogue input channels. Their dynamic range can be adjusted separately and digitization is performed with 8 bit resolution at a $1.25 \mathrm{GHz}$ sampling rate. One channel is connected to the PMT and the remaining channels are connected to the three channels of the SiPM module.

Measurement series In a first measurement, the charge produced by one MIP is determined for the PMT and SiPM. The PMT is operated at a voltage of $1000 \mathrm{~V}$ with a typical gain of $10^{5}$. At lower voltages the MIP signal is not well separated from the noise. For the SiPM, the MIP signal is measured with the HG channel and larger signals are measured with the LG channel.

During the main measurement, a maximum dynamic range of all components in the setup is required, and thus the PMT was operated at $800 \mathrm{~V}$. The conversion for the MIP signal from $1000 \mathrm{~V}$ to $800 \mathrm{~V}$ is done using the datasheet values for the gain ratio. During this measurement only events with signals larger than some hundred MIPs are recorded. Note that due to the low gain of the PMT at $800 \mathrm{~V}$ and the low resolution of the DAQ ( 8 bit), these signals are still affected by the noise.

Results The signals measured with the two detectors are shown in Fig. 4 and an example signal trace is shown in Fig. 5. The expected non-linear behavior of the SiPM response is clearly visible. Nevertheless, the response of the SiPM is fairly linear up to signals involving about $1 / 3$ rd to $1 / 4$ th of the cells, which in this setup corresponds to about 30,000 PE or 1,000 MIP. Throughout the following sections, detected photons (or photons that hit the SiPM) will refer to the number of actual cell-breakdowns caused by incident photons. A typical Photon Detection Efficiency (PDE) of $25 \%$ and illumination with a light pulse of 100,000 photons will thus produce a total of 25,000 detected photons (or hits).

\section{SiPM non-linearity correction}

As shown in Sec. 2, the differences between two devices of identical type are negligible. With an understanding of the SiPM properties in the non-linearity regime a recovery of these signals 


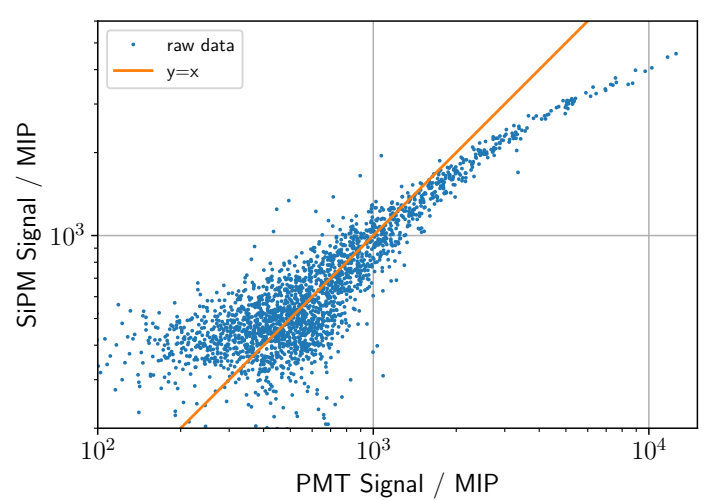

Figure 4: SiPM signal vs. the PMT signal, both calibrated in units of MIP. The deviation from linearity can be seen well for the SiPM signals above $\sim 1,000$ MIP.

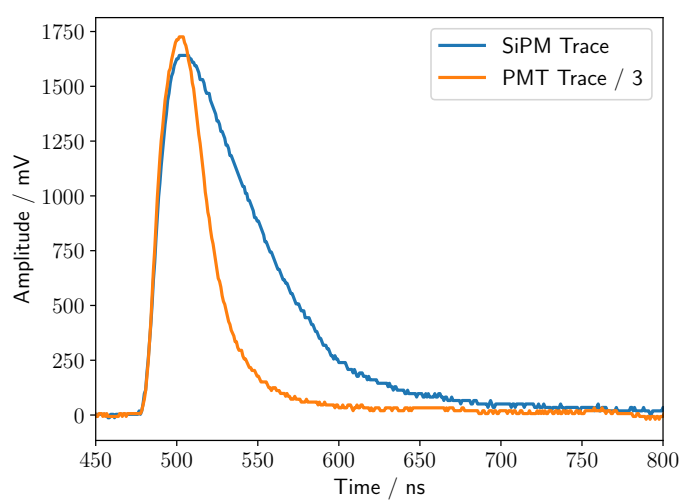

Figure 5: An example of measured traces. The PMT signal is divided by three for better visualization. The SiPM trace has a similar rise time but a longer tail. This particular signal contains $\sim 12,500 \mathrm{MIP}$.

is thus possible without measuring the SiPM characteristics for each device individually. In the following section an algorithm for this recovery is described.

Simulation of a SiPM When an avalanche is induced in a SiPM cell, its charge is released and the cell needs to be recharged. During recharging, PDE, crosstalk, and gain depend on the current state of the charge. An SiPM simulation, which is valid also for large light fluxes that include multiple hits of individual cells, has to take this recharge effects into account. The recharge curve shows two exponential components: for the given type of sensors, the short component has a time constant of $\sim 9 \mathrm{~ns}$ and a long component with a time constant of $\sim 70 \mathrm{~ns}$.

For the simulation of a SiPM, the shape of a response pulse produced by one photon causing an avalanche (single PE) must be known. All larger pulses are obtained by a convolution of the time distribution of photon hits and the single PE response. This measurement has been performed in the lab using the same amplifiers. The pulse is shown in Fig. 6.

The simulation is based on the algorithm published in [9], adapted to correctly describe the saturation with pulses longer than the recharge time. The photons are uniformly distributed over the SiPM cells, for which the times $t_{0}$ of the last photon hits are stored. When the cell is hit again by a photon at a later time $t$, its charge state and the corresponding PDE and gain are calculated. The output corresponds to the released charge. An example of a realistic photon distribution is shown in Fig. 7.

Determination of the photon distribution The goal of the analysis is to determine the amount of photons that hit the SiPM for large light fluxes in a regime of highly non-linear SiPM response.

The analysis is done in two steps: First, an estimate of the photon distribution (orange curve, Fig. 7) is obtained by a deconvolution of the SiPM trace (blue curve, Fig. 5). Second, the SiPM response has to be unfolded from the photon trace (blue curve, Fig. 7).

The measured trace is described by a convolution of the avalanche times and the released charge for one photon (orange curve, Fig. 6). In addition, noise is added to this curve,

$$
s(t)=p(t) * r(t)+n(t),
$$




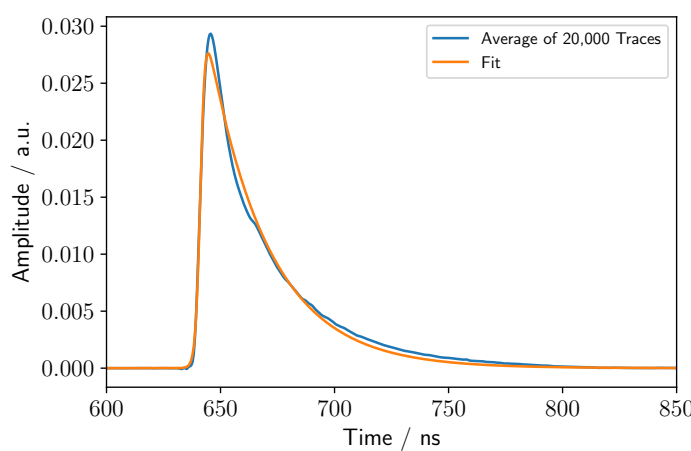

Figure 6: Average SiPM trace measured with a short light pulse $(<200 \mathrm{ps})$ illuminating the SiPM. The fit gives a reasonable approximation of the pulse.

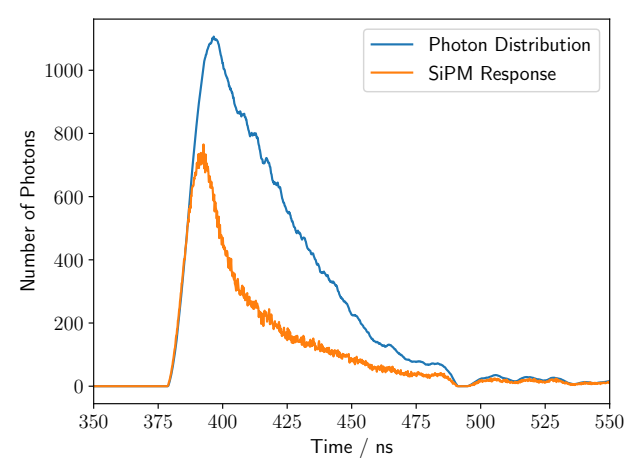

Figure 7: A realistic photon distribution hitting a SiPM. The distribution is already reduced by the PDE so that each photon induces an avalanche. The yellow curve shows input photon distribution (blue) weighted with the SiPM response for each photon.

where $s(t)$ is the measured signal trace, $p(t)$ the photon distribution, $r(t)$ the SiPM response for a single PE, and $n(t)$ random noise. While $r(t)$ and $s(t)$ are known, the noise $n(t)$ has to be estimated from the trace.

The noise is given by the first $320 \mathrm{~ns}$ ( 400 samples) of the measured trace before the signal from the detector. It is extended to have the same length as the whole trace (3000 samples) by taking random samples from these first $320 \mathrm{~ns}$. In addition, the digitization effect is taken into account by adding a uniformly distributed number between 0 and 1 ADC count to each sample.

In order to perform a deconvolution, the whole Eq. (4.1) can be transformed to frequency space by a Fourier analysis,

$$
\hat{s}(f)=\hat{p}(f) \hat{r}(f)+\hat{n}(f),
$$

where $\hat{s}$ describes the Fourier transformation of $s$ and accordingly for the other functions.

An estimate for $\hat{p}$ can be obtained using a deconvolution based on a Wiener filter [10]. It describes a function $\hat{g}$ such that

$$
\hat{p}^{\prime}(f)=\hat{g}(f) \hat{s}(f),
$$

where $\hat{p}^{\prime}$ describes an estimate for $\hat{p}$. Minimizing the expectation for the mean squared distance between $\hat{p}^{\prime}$ and $\hat{p}$ allows to determine $\hat{g}$,

$$
\hat{g}(f)=\frac{1}{\hat{r}(f)} \frac{|\hat{r}(f)|^{2}}{|\hat{r}(f)|^{2}+\hat{N}(f) / \hat{P}(f)} .
$$

Here, $\hat{N}$ and $\hat{P}$ describe the spectral density of the noise and photon distribution to be estimated, respectively. The spectral density for the photon distribution $\hat{P}$ is estimated from the signal $s(t)$ after applying a running mean to reduce the noise contribution. After the Wiener filter, the photon distribution $p(t)$ can be obtained from $\hat{p}^{\prime}(f)$ by a simple inverse Fourier transformation. Fig. 8 shows an example.

Unfolding the SiPM response The photon distribution $p(t)$ described in Sec. 4 still contains the non-linearity of the SiPM. To unfold this non-linearity, the photon distribution $p(t)$ is evaluated 


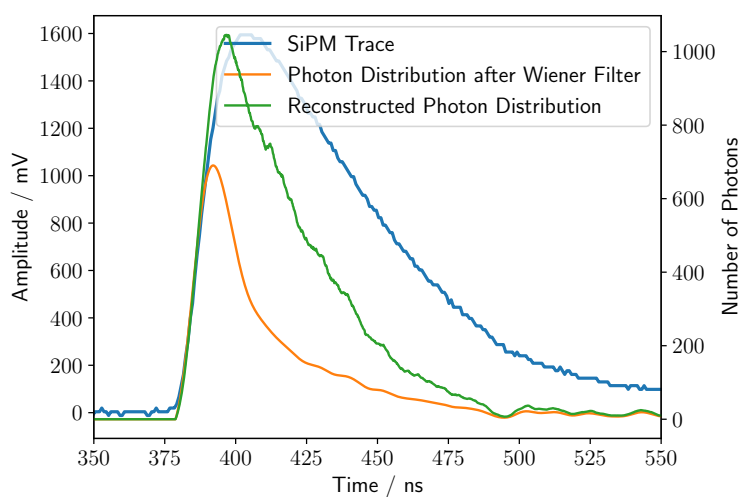

Figure 8: A measured SiPM trace (blue) is shown together with the estimated photon distribution after applying the Wiener deconvolution (orange). In addition, the photon hit distribution for a purely linear response of the sensor is shown (green).

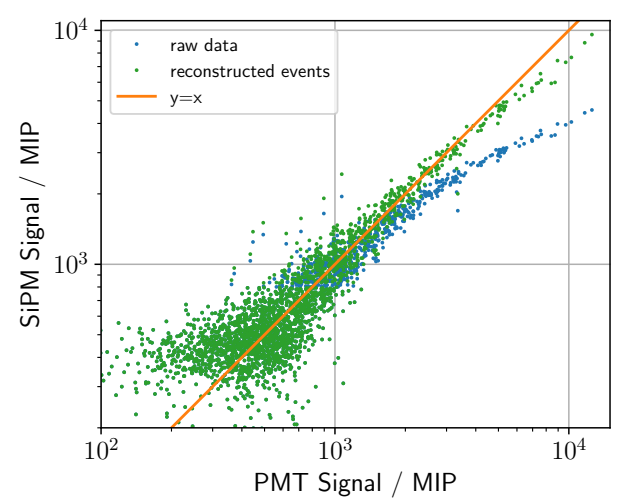

Figure 9: The SiPM signal vs. the PMT signal after applying the reconstruction algorithm. The linearity of the signal is significantly extended.

through the SiPM simulation. The response $\tilde{p}(t)$ is lowered by the non-linearity of the SiPM. The distribution $p(t)$ is then scaled up by the ratio $p(t) / \tilde{p}(t)$ resulting in a new distribution

$$
p_{1}(t)=p(t) \frac{p(t)}{\tilde{p}(t)} .
$$

This procedure is repeated until a distribution $p_{n}(t)$ is obtained such that the response $\tilde{p}_{n}(t)$ is equal to the distribution $p(t)$ after the Wiener deconvolution. The distribution $p_{n}(t)$ is thus an estimate for the true photon distribution that hits the SiPM. Its integral equals to the number of photons that hit the sensor and is proportional to the number of MIP particles traversing the detector. The resulting distribution after applying this algorithm is shown in green in Fig. 8.

Reconstructed events The presented algorithm is applied to all events in the non-linear regime above 800 MIP. The result is shown in Fig. 9. The reconstructed events show a significantly extended linearity range when compared to the raw events and only from $~ 3000$ MIP onwards a deviation from linear behavior can be seen. In Fig. 10 a profile of the relative deviation of the SiPM signal from an ideal linear case is shown. Also here, the improvement due to the reconstruction algorithm is easily observed.

Comparison of two SiPM modules The comparison, described in Sec. 2, shows that no significant difference in the saturation behavior of two SiPMs was observed in the measurements. To study their behavior in a realistic setup, the measurement presented in this proceeding was repeated again with another SiPM module but with the same amplifier board. The serial number of the SiPMs indicate that they are most likely from different production batches and thus from different wafers. For both SiPMs, the same reconstruction algorithm was applied and in Fig. 11 a resulting profile of the ratio of the reconstructed events is shown. While the scatter of the data points is still quite high due to limited statistics for the second SiPM (especially for large signals), the ratios are in a very good agreement. Therefore, signals of both SiPMs can be reconstructed using the same algorithm without measuring the saturation behavior for each SiPM individually. 


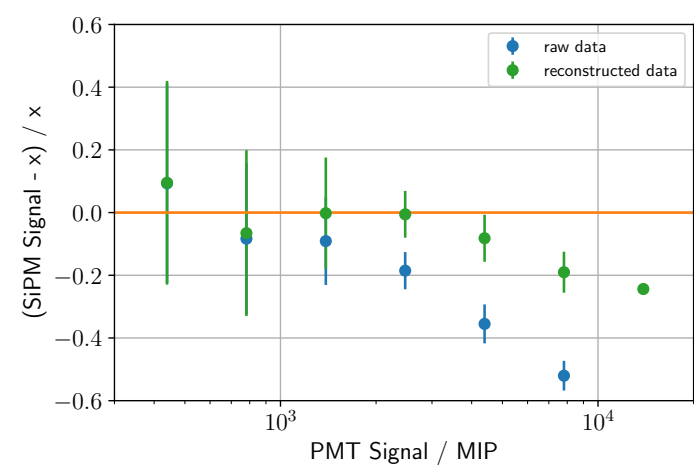

Figure 10: A profile plot of the relative deviation of the SiPM signal to an ideal linear behavior $(x)$. The reconstruction algorithm substantially extends the linear regime of signals.

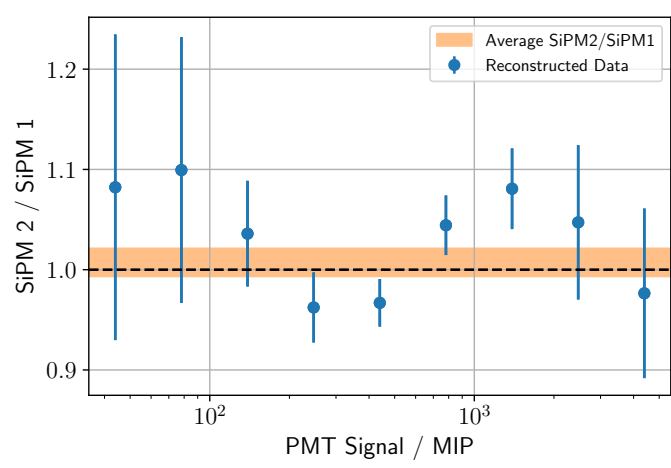

Figure 11: A comparison of two different SiPM modules which shows the their response is in a good agreement.

\section{Conclusions}

In this work, it was demonstrated that two different SiPM devices of the same type show the same response even when operated in the non-linear regime as expected from the high precision of modern semiconductor production. Comparing the signals of two identical co-located scintillator detectors, one read out by a PMT and one by a SiPM, and correcting for the non-linear response of the SiPMs, a good agreement is achieved in the non-linear regime by a reconstruction algorithm described above. No prior knowledge of the photon pulse shape was required.

\section{References}

[1] Hamamatsu Corporation, Hamamatsu S13360 datasheet, June, 2017.

[2] Hamamatsu Corporation, Hamamatsu C11204 datasheet, June, 2017.

[3] R. Winston, J. Miñano and P. Benítez, Nonimaging Optics. Electronics \& Electrical. Elsevier Academic Press, 2005.

[4] Epoxy Technology, Epo-Tek ${ }^{\circledR}$ 310M-2, June, 2017.

[5] Pierre Auger collaboration, A. Aab et al., The Pierre Auger Observatory Upgrade - Preliminary Design Report, 1604.03637.

[6] R. Smida, Scintillator detectors of AugerPrime, PoS ICRC2017 (2017) 390.

[7] Hamamatsu Corporation, Hamamatsu R9420 datasheet, June, 2017.

[8] pico Technology, PicoScope 6000 Series, June, 2017.

[9] FACT collaboration, A. Biland et al., Calibration and performance of the photon sensor response of FACT - The First G-APD Cherenkov telescope, JINST 9 (2014) P10012.

[10] N. Wiener, Extrapolation, interpolation, and smoothing of stationary time series: with engineering applications. Technology Press of the Massachusetts Institute of Technology, 1964. 